\title{
Ações Preventivas contra o Câncer Bucal e Perfil da Doença no Estado da Paraíba
}

\author{
MEIRELES, Sônia Saeger
} COSTA, Lino João da

\begin{abstract}
RESUMO
Esta pesquisa teve por objetivo traçar o perfil dos portadores de câncer bucal, bem como desenvolver ações preventivas contra a doença no Estado da Paraíba. Foram analisados 903 prontuários, sendo 812 arquivados no Hospital Dr. Napoleão Laureano, em João Pessoa/ PB, referentes ao período de 1998 a 2003 e 91 no Centro de Cancerologia Ulisses Pinto, em Campina Grande/ PB, referentes ao período de 1999 a 2002. As palestras foram proferidas em 27 municípios utilizando-se de álbum seriado e folder informativo. Os dados foram coletados, tabulados e analisados através do software SPSS v.10.0. Observou-se que o sexo masculino $(64,5 \%)$ e a faixa etária dos $61-80$ anos $(48,3 \%)$ apresentaram maior prevalência da doença. 0 tipo histológico predominante foi o Carcinoma espinocelular (98\%), acometendo principalmente a língua (31,3\%) e os lábios (24,7\%). O Agreste (20,3\%) e a Mata (19,5\%) foram as macro-regiões do estado com maior número de registros dos casos. Verificou-se que o perfil dos portadores de câncer bucal não sofreu alterações significativas nos últimos anos, bem como falha dos serviços odontológicos nos municípios, tornando-se importante à inclusão do cirurgião-dentista em programas de promoção de saúde, visando à prevenção e diagnóstico do câncer bucal.
\end{abstract}

PALAVRAS-CHAVE:

Câncer bucal. Diagnóstico. Prevenção.

\section{INTRODUÇÃO E REVISÃA DA LITE- RATURA \\ Conforme dados estatísticos do Insti-} tuto Nacional do Câncer (INCA, 2005), o câncer de boca figura como a oitava forma de neoplasia mais freqüente na população brasileira, segundo estimativas para o ano de 2006, onde se esperam 13.470 casos novos $(2,85 \%$ de todos os tumores malignos), tratando-se do $6^{\circ}$ tipo mais comum entre os homens (10.060 novos casos) e o $8^{\circ}$ entre as mulheres (3.410 novos casos) (INCA, 2005).

O câncer bucal é uma doença de etiologia multifatorial, com diversos elementos envolvidos na sua ocorrência (BINNIE; RANKIN; MACKENZIE, 1983). A alta prevalência desta neoplasia está relacionada principalmente ao tabagismo e consumo de bebidas alcoólicas (LOPES et al., 2002; LAM et al., 2006). Outros fatores também podem estar associados, como a história de úlceras orais secundárias à má adaptação de próteses e má higiene oral devido à baixa freqüência de escovações (VELLY et al., 1998; DEDIVITIS et al., 2004).

O Carcinoma espinocelular é o tipo histológico mais freqüente, representando cerca de $90 \%$ a $95 \%$ dos cânceres que ocorrem na boca, sendo maior o risco de desenvolvimento com o aumento da idade, principalmente em homens (BRAN-
DIZZI et al., 2005; LAM et al., 2006). De acordo com Rosa e colaboradores (1997), a característica principal do carcinoma espinocelular é a invasão da lâmina própria e da submucosa pelas células epiteliais, as quais permitem por sua diferenciação, uma graduação histológica de malignidade.

O tratamento do carcinoma espinocelular é feito pela cirurgia, radioterapia ou associação de ambas (REGEZI; SCIUBBA, 2000; NEVILLE et al., 2004). As desvantagens do emprego da radioterapia de forma isolada são o longo período de tratamento e o surgimento de seqüelas posteriores (cáries de irradiação, xerostomia e osteorradionecrose) (KOWALSKI, 1996).

O diagnóstico precoce é bastante dificultado por dois motivos: além de serem assintomáticas, portanto desvalorizadas, as lesões iniciais são raramente identificadas pela maioria dos profissionais no ato do exame clínico.

Este estudo teve por finalidade traçar o perfil dos portadores de câncer bucal, bem como o desenvolvimento de ações preventivas contra a doença no estado da Paraíba.

\section{MATERIAIS E MÉTODOS}

Após a aprovação do Comitê de Ética em Pesquisa do Centro de Ciências da
Saúde da Universidade Federal da Paraíba, iniciou-se a coleta dos dados. $\mathrm{O}$ estudo consistiu numa análise descritiva, de corte transversal. A amostra foi composta por pacientes do ambulatório de cabeça e pescoço de Hospitais de Referência do Estado da Paraíba, no qual foram selecionados os prontuários dos portadores de neoplasias malignas na cavidade bucal (NMCB).

Foram coletadas variáveis referentes ao paciente (sexo, idade e local de residência), a patologia (localização anatômica do tumor, tipo e grau de diferenciação histológica) e ao profissional (encaminhamento hospitalar, tempo de diagnóstico do tumor e conduta terapêutica empregada), a partir da análise dos prontuários registrados no Hospital Dr. Napoleão Laureano em João Pessoa/ PB, relativo aos anos de 1998 a 2003, totalizando 812 casos. No Centro de Cancerologia Ulisses Pinto em Campina Grande/ $\mathrm{PB}$, coletaram-se os dados de 91 prontuários que apresentavam confirmação de neoplasias malignas referentes aos anos de 1999 a 2002, uma vez que este tipo de serviço não era fornecido, anteriormente, na entidade. Em seguida, os dados foram tabulados e analisados com o auxílio do Software SPSS v.10.0.

Ressalta-se que a classificação do grau histológico de malignidade utilizada pelo

\footnotetext{
* Doutoranda em Dentística pela FOUFPel/ RS ** Prof. Adjunto do Departamento de Odontologia Clínica e Social da UFPB/ PB
} 
laboratório da patologia de ambas as instituições seguiu os critérios adotados pela Organização Mundial de Saúde (OMS) (1971), onde o tumor era classificado seguindo uma escala de três graus: grau I ou bem diferenciado, grau II ou moderadamente diferenciado e grau III ou pobremente diferenciado.

Após a coleta e cadastramento dos dados, foram planejadas visitas aos municípios de maior prevalência do Estado da Paraíba, visando, num primeiro momento contactar representantes da administração Municipal e Estadual, através das Secretarias de Saúde, das Associações Comunitárias e outras entidades, para elaboração de estratégias conjuntas. Nas ações foram incluídas palestras abordando aspectos preventivos da doença, orientação sobre o auto-exame de boca e, ao mesmo tempo, distribuição de manuais e boletos informativos neste contexto. Ao final de cada palestra, recolheuse uma lista de presença com o intuito de quantificar a freqüência dos interessados nos trabalhos realizados em cada município.

\section{RESULTADOS}

Foram analisados 903 casos, dos quais $64,5 \%$ eram do sexo masculino e $35,5 \%$ do sexo feminino, observando maior prevalência da doença em homens numa proporção 1,8: 1 .

Pôde-se observar uma redução do número de casos no período de 1999 a 2003 (Tabela 1). A faixa etária mais acometida foi dos 61 aos 80 anos de idade $(48,3 \%)$ (Tabela 2). A localização anatômica de maior prevalência do câncer bucal foi a língua com $31,3 \%$ (283), seguida dos lábios 24,7\% (223), palatos $16,1 \%$ (145), assoalho bucal 10,1\% (91), gengiva $8,6 \%$ (78), região retro-molar $7,6 \%(69)$ e mucosa jugal 1,6\% (14).

Ao exame histopatológico detectou-se alta prevalência do carcinoma espinocelular (98\%), em relação aos outros tipos histológicos. Quanto ao grau de diferenciação histológica, pôde-se observar maior freqüência do padrão moderadamente diferenciado $(55,3 \%)$ (Tabela 3$)$.

Observou-se ausência de informações em número elevado de prontuários (Tabelas 4, 5 e 6) o que não nos permite extrapolar os dados obtidos para as seguintes referências: encaminhamento hospitalar, tempo de diagnóstico do tumor e conduta terapêutica empregada, respectivamente.

Segundo os dados levantados nas entidades hospitalares de João Pessoa e Campina Grande, a macro-região do Estado com maior prevalência de indivíduos afetados foi o Agreste com 20,3\%
(183), seguida da Zona da Mata 19,5\% (176), Sertão 17,7\% (160) e Borborema $3,8 \%$ (34). Em 2\% (18) dos casos os pacientes foram provenientes de outros estados, e, em 36,8\% (332) dos prontuários esta informação não estava disponível.

Quanto às ações preventivas contra o câncer bucal, cerca de 785 pessoas foram diretamente beneficiadas com as palestras realizadas nos 27 municípios durante a campanha, além daquelas favorecidas através dos debates e entrevistas realizadas nas Rádios Comunitárias em alguns municípios.

\section{DISCUSS ÃO}

$A$ análise dos dados nos permite afirmar que a doença ainda predomina no sexo masculino (1,8: 1), no entanto, há uma tendência ao equilíbrio desta proporção, principalmente quando se constata a modificação de hábitos e costumes das mulheres, as quais estão se expondo com uma maior freqüência aos fatores considerados de risco, como o uso do álcool, do fumo e exposição à radiação solar (ELIAS et al., 2002; RAWASHDEH; MATALKA, 2004). A meta-análise realizada por Brandizzi e colaboradores (2005) revisou os estudos mais relevantes sobre o câncer bucal publicados na cidade de Buenos Aires/ Argentina. Após a análise do total de casos, correspondentes ao período de 1950-2000, os autores concluíram que a prevalência do câncer bucal no sexo feminino tem aumentado significativamente.

Rawashdeh; Matalka, (2004), realizaram um estudo retrospectivo no norte da Jordânia a fim de determinar as principais características dos portadores de neoplasias malignas da cavidade oral no período de 1991-2001. Os autores observaram que 125 pacientes apresentavam diagnóstico de tumor maligno primário, dos quais $64 \%$ eram do sexo masculino e $36 \%$ do feminino. A proporção observada foi de 1,77:1 para o sexo masculino, corroborando com os nossos achados. No entanto, ainda há divergência entre os resultados encontrados na literatura e, estes podem ser influenciados por uma série de variáveis como regiões geográficas, hábitos e costumes, condições sociais e culturais da população.

Em relação à idade, encontramos maiores freqüiências dos tumores nos indivíduos com faixa etária de 41 a 80 anos, assemelhando-se com os demais estudos de Kerdpon e Sriplung (2001), Lam et al. (2006). O câncer é considerado uma doença que tem incidência crescente, aumentando nas faixas etárias mais elevadas, tendo seu pico na sexta e sétima dé- cadas de vida (NEVILLE et al., 2004; RAWASHDEH; MATALKA, 2004).

Os resultados mostraram que a língua foi à região anatômica de maior ocorrência de câncer bucal. Estes dados poderão variar de estudo para estudo. Lopes e colaboradores (2002) citam os lábios $(39,02 \%)$ e a língua $(18,7 \%)$ como localizações anatômicas mais acometidas. A língua é o local da cavidade oral mais comumente afetado pelo tumor, seguida dos lábios, principalmente o inferior, devido a maior exposição solar (PARISE JÚNIOR, 2000; RAWASHDEH; MATALKA, 2004).

O carcinoma espinocelular foi o tipo histológico mais freqüente $(98 \%)$, sendo este dado coerente com a unanimidade dos autores Elias et al. (2002), Dedivitis et al. (2004), Rawashdeh e Matalka (2004). Quanto ao grau de diferenciação histológico, o tipo moderadamente diferenciado mostrou-se mais prevalente, seguido do bem diferenciado. Em contrapartida, em análise realizada por Rosa e colaboradores (1997) pôde-se constatar que o tipo bem diferenciado foi o mais ocorrente (75\%). Regezi e Sciubba (2000) relatam que a maioria dos carcinomas espinocelulares é de lesões bem ou moderadamente diferenciadas. $\mathrm{O}$ grau histopatológico do tumor está relacionado ao seu comportamento biológico, ou seja, um tumor maduro o suficiente para parecer com o tecido de origem parece crescer mais lentamente e metastatizar mais tardiamente (NEVILLE et al., 2004).

A macro-região do estado da Paraíba que apresentou maior freqüência da doença foi o Agreste, seguido da Mata e Sertão. Em estudo realizado por Barreto e colaboradores (2001) a macro-região do estado da Paraíba com maior prevalência do câncer bucal foi o Litoral (39,9\%), seguido do Agreste $(27,2 \%)$ e Sertão $(25,9 \%)$. Possivelmente, a exposição crônica à radiação solar pôde atuar como um potencial fator de risco para o aumento do número de casos da doença nestas regiões do estado.

A ausência de informações numa taxa elevada dos prontuários não nos permitiu realizar uma análise detalhada dos dados referentes ao encaminhamento hospitalar, tempo de diagnóstico da lesão e conduta terapêutica empregada. Brito e colaboradores (1998) relatam que o não registro, por escrito, de informações importantes nos prontuários, deve ser objeto de um processo de conscientização dos oncologistas e demais profissionais em saúde, tornando-se necessário à realização de treinamentos sistemáticos para minimizações dessas dificuldades.

Os fatores relacionados ao diagnósti- 
co tardio das NMCB não estão suficientemente esclarecidos. Estudos relatam que o atraso no diagnóstico pode estar associado tanto a características inerentes ao paciente (KERDPON; SRIPLUNG, 2001) quanto ao profissional (ONIZAWA et al., 2003). Leão e colaboradores (2005) avaliaram o conhecimento e competência clínica dos cirurgiõesdentistas de Recife-PE sobre o câncer bucal e verificaram que os mesmos não apresentavam uma adequada compreensão sobre as causas comuns e características clínicas da doença. Em outro estudo, Hayassy (1998) verificou que $70 \%$ dos cirurgiões-dentistas da rede pública de Niterói-RJ não estavam capacitados a prevenir o câncer bucal, devido à insuficiência de conhecimentos específicos.

Faz-se necessário à reciclagem profissional através de cursos contínuos de atualização, enfatizando o diagnóstico e tratamento tanto das manifestações iniciais quanto das lesões cancerizáveis (KERDPON; SRIPLUNG, 2001; LEÃO et al., 2005). A prevenção e diagnóstico precoce são, sem dúvida, as medidas mais eficazes para a melhoria do prognóstico do câncer, significando maior possibilidade de cura, fazendo com que o tratamento seja menos mutilante e mais eficiente (NOORMAN VAN DER DUSSEN, 1994).

Durante a realização da campanha preventiva, pôde-se comprovar falha dos serviços odontológicos nos municípios, tornando-se importante à inclusão do cirurgião-dentista em programas de promoção de saúde, visando à prevenção e diagnóstico do câncer bucal. Segundo Dedivitis e colaboradores (2004) estratégias de educação dos pacientes e profissionais de saúde para o diagnóstico precoce e encaminhamento para tratamento imediato contribuiriam para a melhoria do serviço e do prognóstico para os pacientes portadores do carcinoma espinocelular.

\section{CONCLUS $\tilde{A} O$}

Dentro das limitações deste estudo, pôde-se concluir que o perfil do portador de câncer bucal do Estado da Paraíba é do gênero masculino, com faixa etária superior aos 60 anos e as regiões anatômicas de maior prevalência continuam sendo a língua e os lábios. Grande parte dos portadores acometidos pela neoplasia provinha do Agreste e Zona da Mata do estado.

\section{AGRADECIMENTOS}

Os autores agradecem a COLGATE pelo incentivo a pesquisa e o desenvolvimento de ações preventivas entre os aca- dêmicos de Odontologia do país, por subsídio do "4" Prêmio Nacional de Odontologia Preventiva -COLGATE", onde este trabalho foi premiado como terceiro colocado na categoria pesquisa.e parte dos portadores acometidos pela neoplasia provinha do Agreste e Zona da Mata do estado.

\section{AGRADECIMENTOS}

Os autores agradecem à COLGATE pelo incentivo a pesquisa e o desenvolvimento de ações preventivas entre os acadêmicos de Odontologia do país, por subsídio do "4 4 " Prêmio Nacional de Odontologia Preventiva -COLGATE", onde este trabalho foi premiado como terceiro colocado na categoria pesquisa.

\section{ABSTRACT}

The aim of this study was traced the profile of oral cancer and also developing preventive actions against this disease in Paraíba state. There were analyzed 903 patients' promptuaries, which 812 were filed in Dr. Napoleão Laureanós Hospital (João Pessoa/ PB) referred to the period of 1998 to 2003 , and 91 files in Cancerologia Ulisses Pinto's Center (Campina Grande/PB) referred to the period of 1999 the 2002 . The lectures had been pronounced in 27 cities using an illustrated specific album and informative folders. The data had been collected, tabulated and analyzed through software SPSS v.10.0. It was observed that men $(64,5 \%)$ and people between 61 to 80 years of age $(48,3 \%)$ had presented highest prevalence of the studied disease. The most frequent histological type was the squamous cell carcinoma (98\%), mainly compromising the tongue $(31,3 \%)$ and the lips $(24,7 \%)$. The Agreste (20,3\%) and Mata $(19,5 \%)$ had been the macro-regions of the state with the highest cases records. This study verified that the profile of oral cancer did not suffer significant alterations in the last years as well it confirmed the imperfection of the dentistry services in the cities becoming important the inclusion of the dentists in programs of health promotion, aiming the prevention and diagnosis of oral cancer.

\section{KEY-WORDS:}

Oral cancer. Diagnostic. Prevention.

\section{REFERÊNCIAS}

BARRETO, R.C. et al. Paraíba: Uma das Maiores Prevalências de Câncer Bucal da Federação Brasileira. Conceitos, v.4, n.6, p.118-122, 2001.
BINNIE, W.H.; RANKIN, K.V.; MACKENZIE, I.C. Etiology of Oral Squamous Cell Carcinoma. J. Oral. Pathol., v.12, n.1, p.11-29, Feb. 1983.

BRANDIZZI, D. et al. Analysis of the Epidemiological Features of Oral Cancer in the City of Buenos Aires. Acta. Odontol. Latinoam., v.18, n.1, p.31-35, 2005.

BRITO, A.M.S. Reimplantação do Serviço de Câncer de Base Populacional em Pernambuco, 1995 e 1996. In: Congresso Brasileiro de Epidemiologia, 4, 1998. Anais IV Congresso Brasileiro de Epidemiologia, Recife: 1998. Resumo 127, p. 176 .

DEDIVITIS, R.A. et al. Características Clinico-epidemiológicas no Carcinoma Espinocelular de Boca e Orofaringe. Rev. Bras. Otorrinolaringol., v.70, n.1, p.35-40, 2004.

ELIAS, R. et al. Câncer Bucal. J. Bras. Clin. Odontol., v.6, n.31, p.25-28, 2002 .

HAYASSY, A. Câncer Bucal no Setor Público de Saúde. Rev. Bras. Odontol. v.55, n.3, p.173-175,1998.

INSTITUTO NACIONAL DO CÂNCER. Ministério da Saúde. Instituto Nacional do Câncer. Estimativa 2006 - Incidência de Câncer no Brasil. Rio de Janeiro, 2005.

KERDPON, D.; SRIPLUNG, H. Factors Related to Delay in Diagnosis of Oral Squamous Cell Carcinoma in Southern Thailand. Oral. Oncology., v. 37, n.2, p. 127-131, 2001.

KOWALSKI, L.P. Manual de Condutas Diagnósticas e Terapêuticas em Oncologia. São Paulo: Âmbito Editores, 1996.

LAM, L.; LOGAN, R.M.;LUKE, C. Epidemiological Analysis of Tongue Cancer in South Australia for the 24-Year Period, 1977-2001. Aust. Dent. J., v.51, n.1, p.16-22, 2006.

LEÃO, J.C. et al. Knowledge and Clinical Expertise Regarding Oral Cancer Among Brazilian Dentists. Int. J. Oral Maxillofac. Surg., v.34, n.4, p.436-439, 2005. 
LOPES, F.F. et al. Aspectos Epidemiológicos e Terapêuticos do Câncer Bucal. Rev. Bras. Odontol., v.59, n.2, p.98-99, 2002.

NEVILLE, B. W. et al. Patologia Oral e Maxilofacial. 2.ed. Rio de Janeiro: Guanabara Koogan, 2004.

NOORMAN VAN DER DUSSEN, M.F. Oral Cancer. The Importance of Early Diagnosis and Role of the Dentist. Rev. Belge. Med. Dent., v.49, n.1, p.35-49, 1994.

ONIZAWA, K. et al. Factors Associated with Diagnostic Delay of Oral Squamous Cell Carcinoma. Oral. Oncology., v. 39, n. 8, p.781-788, 2003.

PARISE JUNIOR, O. Câncer de Boca: Aspectos Básicos e Terapêu-

Tabela 1: Distribuição dos casos segundo o ano em que foram diagnosticadas.

\begin{tabular}{ccc}
\hline Ano de ocorrência & n & $\%$ \\
\hline $\mathbf{1 9 9 8}$ & 175 & 19,4 \\
$\mathbf{1 9 9 9}$ & 181 & 20 \\
$\mathbf{2 0 0 0}$ & 136 & 15,1 \\
$\mathbf{2 0 0 1}$ & 148 & 16,4 \\
$\mathbf{2 0 0 2}$ & 144 & 15,9 \\
$\mathbf{2 0 0 3}$ & 119 & 13,2 \\
Total & 903 & 100
\end{tabular}

Tabela 4: Distribuição dos casos segundo encaminhamento hospitalar dos portadores.

\begin{tabular}{ccc}
\hline $\begin{array}{c}\text { Encaminhamento } \\
\text { hospitalar }\end{array}$ & $\mathbf{n}$ & $\%$ \\
\hline Médico & 375 & 41,5 \\
Cirurgióes-Dentistas & 6 & 0,7 \\
Espontâneo & 23 & 2,5 \\
Outros & 34 & 3,8 \\
Não informado & 465 & 51,5 \\
Total & 903 & 100 \\
\end{tabular}

ticos. São Paulo: Sarvier Ltda, 2000.

RAWASHDEH, M.A.; MATALKA, I. Malignant Oral Tumors in Jordanians, 1991-2001. A Descriptive Epidemiological Study. Int. J. Oral Maxillofac. Surg., v.33, p. 183-188, 2004.

REGEZI, J.A.; SCIUBBA, J.J. Patologia Bucal - Correlações Clínicopatológicas. Rio de Janeiro: Guanabara Koogan, 2000.

ROSA, M.R.D.; FALCÃO, A.V.; SAMPAIO, F.G. Epidemiologia do Câncer Bucal no Estado da ParaíbaBrasil (1978-1993). Rev. Bras. Cien. Saúde., v.1, n.1/3, p.17-22, 1997.

VELLY, A.M. et al. Relationship Between Dental Factors and Risk of Upper Aerodigestive Tract Cancer. Oral. On-

Tabela 2: Distribuição dos casos se gundo a faixa etária dos portadores.

\begin{tabular}{ccc}
\hline Faixa etária & n & $\%$ \\
\hline 0-20 & 4 & 0,4 \\
$\mathbf{2 1 - 4 0}$ & 45 & 5 \\
$\mathbf{4 1 - 6 0}$ & 272 & 30,1 \\
61-80 & 436 & 48,3 \\
Acima de 80 & 117 & 13 \\
Não informado & 29 & 3,2 \\
Total & 903 & 100
\end{tabular}

Tabela 5: Distribuição dos casos segundo o tempo de diagnóstico.

\begin{tabular}{ccc}
\hline Tempo de diagnóstico & $\mathbf{n}$ & $\%$ \\
\hline Até 3 meses & 171 & 18,9 \\
$\mathbf{3}$ a 6 meses & 140 & 15,5 \\
6 meses a 1 ano & 125 & 13,8 \\
Acima de 1 ano & 71 & 7,9 \\
Não informado & 396 & 43,9 \\
Total & 903 & 100
\end{tabular}

cology., v.34, n.4, p.284-291, 1998.

WHO. Histological Typing of Oral and Oropharyngeal Tumours. International Histological Classi.cation of Tumours, n. 4, Geneva: WHO, 1971.

Endereço para correspondência

* Autora responsável pela correspondência

Universidade Federal de Pelotas/ Faculdade de Odontologia

R Gonçalves Chaves, 457 - $5^{\circ}$ andar. CEP.: 96015-560 Centro Pelotas/ RS Email: soniasaeger@hotmail.com

** Rua Dr. João Franca, 242 apt $^{\circ}$ 1101

CEP.: 50038-190 - Manaíra - João Pessoa/ PB

Email: linoj@uol.com.br

Tabela 3: Distribuição dos casos segundo o tipo de diferenciação histológico.

\begin{tabular}{ccc}
\hline Grau histológico & $\mathbf{n}$ & $\%$ \\
\hline CEC GI & 303 & 34,2 \\
CEC GII & 489 & 55,3 \\
CEC GIII & 61 & 6,9 \\
Grau não informado & 31 & 3,5 \\
Total & $\mathbf{8 8 4}$ & $\mathbf{1 0 0}$
\end{tabular}

Tabela 6: Distribuição dos casos segundo a conduta terapêutica empregada.

\begin{tabular}{ccc}
\hline $\begin{array}{c}\text { Conduta } \\
\text { terapêutica empregada }\end{array}$ & $\mathbf{n}$ & $\%$ \\
\hline Cirurgia & 191 & 21,2 \\
Radioterapia & 132 & 14,6 \\
Quimioterapia & 8 & 0,9 \\
Cirurgia + Radioterapia & 136 & 15,1 \\
Outras condutas & 39 & 4,3 \\
Não informado & 397 & 44 \\
Total & 903 & 100
\end{tabular}

